\title{
Bilateral transient osteoporosis of the knee during pregnancy
}

\author{
Charpidou, Theodosia ; Lang, Franziska ; Langenegger, Thomas ; Dedes, Konstantin J ; Honegger, \\ Christoph
}

\begin{abstract}
Transient osteoporosis is a rare disease, which can affect pregnant women in the third trimester. Bone marrow edema syndrome, transient bone demineralization, hip algodystrophy, regional migratory osteoporosis, and even reflex sympathetic dystrophy all are terms that have been used to describe the condition. Babinsky and Fromen first documented the symptoms of transient osteoporosis in 1916, and in 1959 Curtiss and Kincaid discussed two cases affecting women in the third trimester of pregnancy. The hip joints are most commonly affected in pregnant women, being reported in $76 \%$ of cases.
\end{abstract}

DOI: https://doi.org/10.1007/s00404-012-2628-4

Posted at the Zurich Open Repository and Archive, University of Zurich ZORA URL: https://doi.org/10.5167/uzh-74312

Journal Article

Published Version

Originally published at:

Charpidou, Theodosia; Lang, Franziska; Langenegger, Thomas; Dedes, Konstantin J; Honegger, Christoph (2013). Bilateral transient osteoporosis of the knee during pregnancy. Archives of Gynecology and Obstetrics, 287(6):1259-1261.

DOI: https://doi.org/10.1007/s00404-012-2628-4 


\title{
Bilateral transient osteoporosis of the knee during pregnancy
}

\author{
Theodosia Charpidou • Franziska Lang • \\ Thomas Langenegger $\cdot$ Konstantin J. Dedes • \\ Christoph Honegger
}

Received: 29 April 2012/Accepted: 5 November 2012/Published online: 21 November 2012

(C) Springer-Verlag Berlin Heidelberg 2012

\section{Introduction}

Transient osteoporosis is a rare disease, which can affect pregnant women in the third trimester [1-5]. Bone marrow edema syndrome, transient bone demineralization, hip algodystrophy, regional migratory osteoporosis, and even reflex sympathetic dystrophy all are terms that have been used to describe the condition [6].

Babinsky and Fromen first documented the symptoms of transient osteoporosis in 1916, and in 1959 Curtiss and Kincaid [7] discussed two cases affecting women in the third trimester of pregnancy. The hip joints are most commonly affected in pregnant women, being reported in $76 \%$ of cases [6].

\section{Case report}

A 39-year-old Caucasian woman, in the 39th week of pregnancy with gravida 3 , para 1 presented with a 6 -week history of pain in the right knee, followed by an episode of pain in the left knee. The course of the pregnancy was otherwise

T. Charpidou $\cdot$ F. Lang $\cdot$ K. J. Dedes $(\bowtie) \cdot$ C. Honegger Department of Gynecology and Obstetrics, Zuger Kantonsspital AG, 6340 Baar, Switzerland

e-mail: konstantin.dedes@usz.ch

T. Charpidou

e-mail: charpidou@gmail.com

F. Lang

e-mail: franziska.lang@zgks.ch

C. Honegger

e-mail: christoph.honegger@zgks.ch

T. Langenegger

Department of Rheumatology, Zuger Kantonsspital,

Baar, Switzerland

e-mail: Thomas.langenegger@zgks.ch uneventful. There was no previous history of joint problems, alcohol excess, corticosteroid or heparin use, and trauma or acute medical illness. The right knee deteriorated over 2-3 weeks, followed by pain in the left knee until she was unable to walk. Clinical examination revealed tenderness over the femoral condyle and the tibia, and limitation of bilateral knee movements due to pain. There was no effusion. Analgesics did not improve the symptoms and therefore labor was induced in the 39th week of pregnancy.

Postpartum serum white cell count and C-reactive protein levels were within normal limits. The erythrocyte sedimentation rate was slightly raised at $54 \mathrm{~mm} / \mathrm{h}$. Creatinine, electrolytes, thyroid hormone, and liver function tests were all within normal range. Tests for antinuclear antibodies and Borrelia serology were negative.

Magnetic resonance imaging (MRI) showed decreased signal intensity on T1-weighted images on the right knee, especially within the lateral tibia surface, and the lateral femoral condyle. Correspondingly, the signal intensity on T2-weighted images was increased over the same area, consistent with extensive edema of the right knee. In the left knee, there was a moderate amount of edema and increased signal intensity on T2-images in the lateral femoral condyle, although less intense than on the right.

Three weeks after labour and under paracetamol and ibuprofen, pain receded slightly. The pain disappeared almost completely after two months.

On the 2-month follow-up, the clinical symptoms had almost resolved. MRI showed no more signal abnormalities in the knees.

\section{Discussion}

The etiology of idiopathic transient osteoporosis in pregnancy remains unknown. The most common theory is that 
microvascular injury causes tissue ischemia, bone marrow edema, and limited cell injury of fat and hematopoietic cells, but not osteocytes $[6,8,9]$. There is also a reported case of transient osteoporosis involving the knee following trauma [9]. Additionally, there is a hypothesis that regional transient osteoporosis may be associated with vitamin C deficiency [10]. Carbone et al. [11] suggested that transient osteoporosis in pregnancy may be due to genetic predisposition.

Although transient osteoporosis was originally described in pregnant women, young or middle-aged men are more commonly affected [12]. The onset of pain is usually spontaneous, and comes without a history of trauma. Pain can range from mild to debilitating, depending on the stage of the disease. Upon examination, an effusion may be observed. Tenderness to palpation is present at the femoral
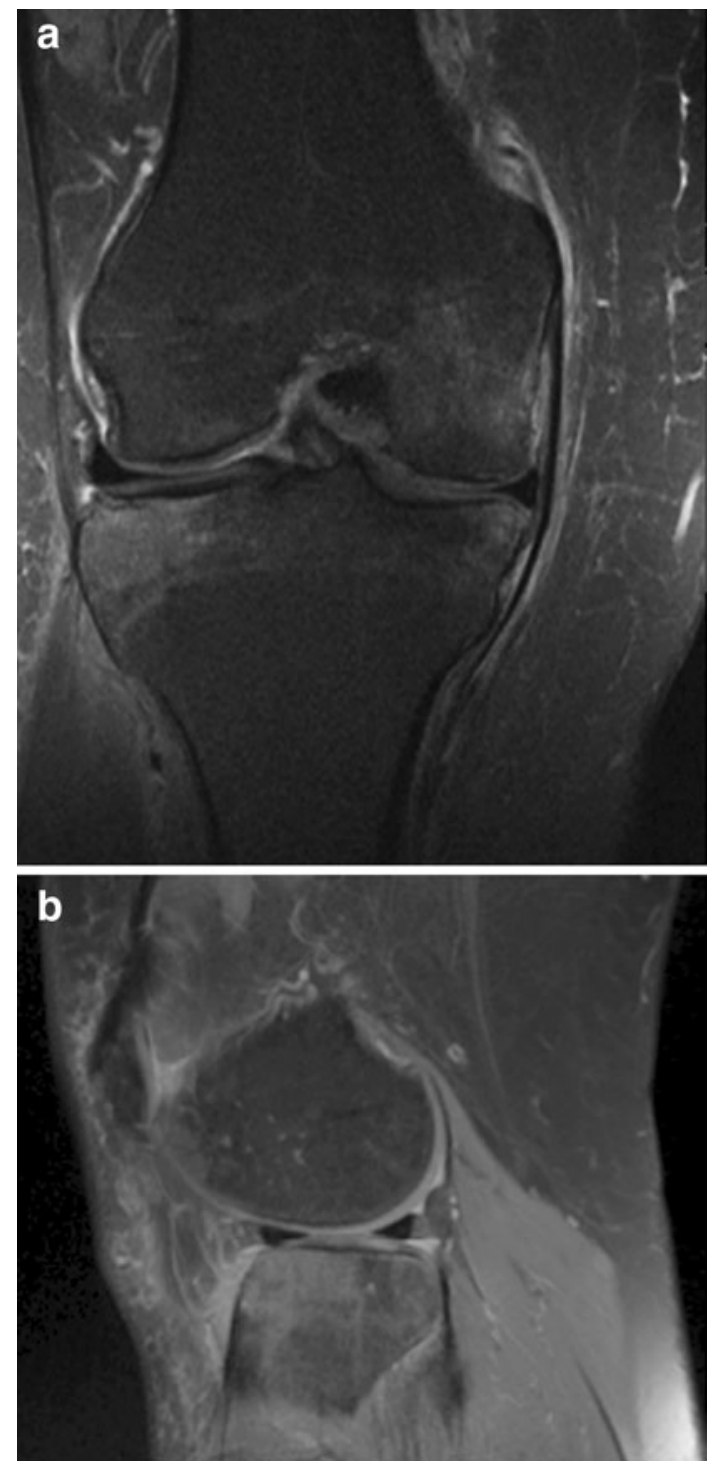

Fig. 1 Right knee: diffuse areas of increased signal in the lateral tibia surface and the medial femoral condyle: a coronal, b sagittal condyle and tibio-femoral joint. This finding is often misleading-indicating meniscal or articular surface injury. Laboratory values are usually normal or non-specific. Clinical improvement occurs over weeks to months, without specific therapy [6].

Plain radiographs in cases of transient osteoporosis may show normal findings initially, but patients often develop a profound osteopenia within several weeks. Skeletal scintigraphy may show increased tracer uptake prior to the development of osteopenia on plain radiographs. MRI shows a diffuse area of decreased signal intensity on T1-weighted images and increased signal intensity on $\mathrm{T} 2$-weighted images that is typical for osteonecrosis [13-15].
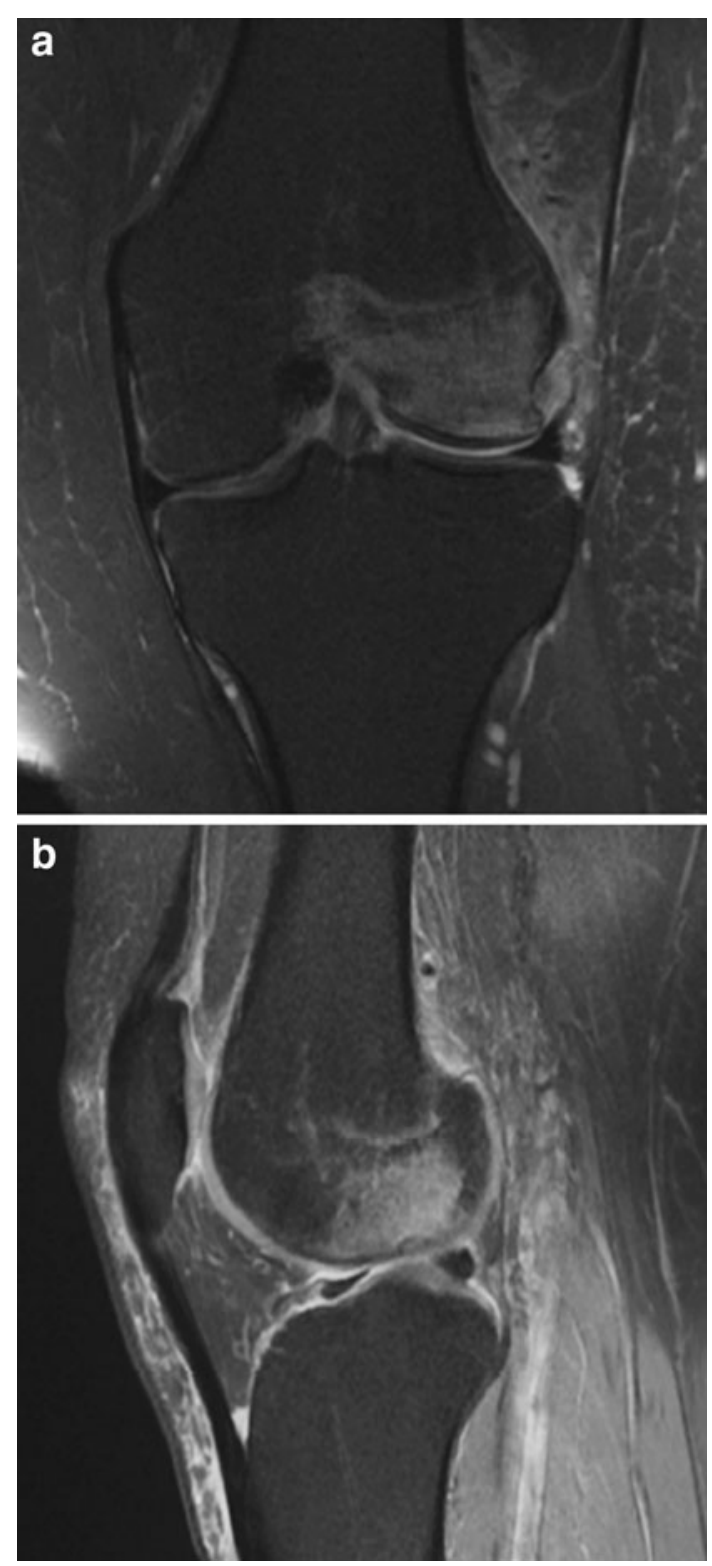

Fig. 2 Left knee: diffuse area of increased signal intensity in the lateral femoral condyle: a coronal, b sagittal 
Treatment typically consists of a conservative approach: limiting weight bearing and avoiding activities that aggravate pain. Pain killers should also be avoided. Several additional forms of treatment have been described, including corticosteroids (prednisone up to $40 \mathrm{mg} /$ day), bisphosphonates, calcitonin treatment, and calcium substitution up to $2 \mathrm{~g} /$ day $[6,11,16]$.

Transient osteoporosis during pregnancy is a rare, selflimiting condition, typically appearing during the third trimester. Therefore, we recommend a conservative approach to treatment. This includes the administration of analgesics, bimonthly follow-ups, and a recommendation to the patient to restrict weight-bearing.

To date, the hip is the most common location for transient osteoporosis [17]. The knees or ankles are far less commonly affected, and very few cases have been reported $[18,19]$. Only two other documented cases have been reported, where both knees were affected [3, 20]. Musculoskeletal complaints by the mother during pregnancy are very common, and although transient osteoporosis is a rare disease, it should be considered as a possible differential diagnosis (Figs. 1a, b, 2a, b).

Conflict of interest None.

\section{References}

1. Beaulieu JG, Razzano CD, Levine RB (1976) Transient osteoporosis of the hip in pregnancy. Clin Orthop Relat Res 115: 165-168

2. Smith R et al (1985) Osteoporosis of pregnancy. Lancet 1(8439):1178-1180

3. Stamp L et al (2001) Bilateral transient osteoporosis of the knee in pregnancy. Ann Rheum Dis 60(7):721-722
4. Fazekas JE et al (2002) Migratory transient osteoporosis of the hip occurring before and during pregnancy. J Women Imag $4(2): 80-85$

5. Garcia Renedo RJ et al (2010) Acute knee pain in pregnancy. Case report of regional transient osteoporosis. Rheumatol Clin 6(2):99-101

6. Nikolaou VS et al (2008) Transient osteoporosis of the knee. Orthopedics 31(5):502

7. Curtiss PH Jr, Kincaid WE (1959) Transitory demineralization of the hip in pregnancy. A report of three cases. J Bone Joint Surg Am A 41:1327-1333

8. Clemetson IA et al (2004) Postpartum osteoporosis associated with proximal tibial stress fracture. Skeletal Radiol 33(2):96-98

9. El Masry MA, Saha A, Calder SJ (2005) Transient osteoporosis of the knee following trauma. J Bone Joint Surg Br 87(9):1272-1274

10. Rodriguez $\mathrm{S}$ et al (2007) Regional transient osteoporosis of the foot and vitamin C deficiency. Clin Rheumatol 26(6):976-978

11. Carbone LD et al (1995) Osteoporosis of pregnancy: long-term follow-up of patients and their offspring. Obstet Gynecol 86(4 Pt 2): 664-666

12. Parker RK, Ross GJ, Urso JA (1997) Transient osteoporosis of the knee. Skeletal Radiol 26(5):306-309

13. Bloem JL (1988) Transient osteoporosis of the hip: MR imaging. Radiology 167(3):753-755

14. Hauzeur JP et al (1991) Study of magnetic resonance imaging in transient osteoporosis of the hip. J Rheumatol 18(8):1211-1217

15. Wilson AJ et al (1988) Transient osteoporosis: transient bone marrow edema? Radiology 167(3):757-760

16. Lakhanpal S et al (1987) Transient regional osteoporosis. A study of 56 cases and review of the literature. Ann Intern Med 106(3):444-450

17. Willis-Owen CA et al (2008) Bilateral femoral neck fractures due to transient osteoporosis of pregnancy: a case report. Cases J $1(1): 120$

18. Grey A, Dalbeth N, Doyle A (2009) Clinical images: transient regional osteoporosis. Arthritis Rheum 60(10):3145

19. Lloyd JM, Lewis M, Jones A (2006) Transient osteoporosis of the knee in pregnancy. J Knee Surg 19(2):121-123

20. Ververidis AN et al (2009) Bilateral transient bone marrow edema or transient osteoporosis of the knee in pregnancy. Knee Surg Sports Traumatol Arthrosc 17(9):1061-1064 